\title{
SAÚDE MENTAL EM SITUAÇÃO DE EMERGÊNCIA: COVID-19
}

\section{MENTAL HEALTH IN AN EMERGENCY SITUATION: COVID-19}

\section{Resumo}

Este texto traz reflexões e orientações técnicas e científicas frente à pandemia do novo coronavírus, uma situação de crise e emergência com reflexos sociais, econômicos e na saúde das populações e dos profissionais expostos diretamente aos riscos de contaminação. O foco principal são os problemas de saúde mental, tendo em vista o temor pela exposição ao contágio, a situação de isolamento e confinamento e a medida de quarentena adotada em vários países. São discutidas em evidências científicas e inferências produzidas na literatura nacional e internacional sobre os impactos da COVID-19 na saúde das pessoas, especialmente as mais vulneráveis, e as possibilidades de contenção, mitigação e prevenção de sintomas de transtornos mentais. Nesse contexto, apontam-se os impactos na saúde mental observados e previstos nos profissionais que atuam na linha de frente da situação de emergência.

Palavras-chave: Emergências, saúde mental, pandemias.

\section{Abstract}

This text presents reflections and technical and scientific guidance on the COVID-19 outbreak, a critical emergency situation with social and economic repercussions and implications for the health of populations and of the professionals directly exposed to contamination risks. The main focus of this paper is on the mental health issues involved, considering fear of exposure to the virus and the isolation and confinement policies and quarantine procedures adopted in several countries. Discussions are based on the scientific evidence and inferences presented in Brazilian and international literature with relation to the impacts of COVID-19 on people's health, especially the most vulnerable groups, and the possibilities for containing, mitigating, and preventing symptoms of mental disorders. Both observed and predicted impacts on the mental health of professionals working on the front line of the emergency situation are discussed.

Keywords: Emergencies, mental health, pandemics.

Este texto trata de reflexões, orientações e suporte técnico-científico às práticas da psicologia e da medicina no âmbito da gestão da crise produzida pela pandemia do coronavírus, tal como foi declarada oficialmente pela Organização Mundial da Saúde (OMS) em 11 de março de 2020. O surto do coronavírus de 2019 (coronavirus disease of 2019 - COVID-19), síndrome respiratória aguda grave (severe acute respiratory syndrome - SARS-CoV-2) ou pneumonia de Wuhan, como foi declarado pela OMS em janeiro de 2020, tornou-se uma emergência de saúde pública e de interesse internacional ${ }^{1,2}$. À medida que 0 coronavírus se espalha nas diferentes regiões do mundo, inclusive no Brasil, políticas de saúde pública acentuam a necessidade de contenção e isolamento, que se dirigem, também, para o atraso e/ou mitigação da velocidade de difusão da doença, com forte envolvimento em ações de prevenção à contaminação ${ }^{3,4}$. Em alguns casos isolados, como no Reino Unido, as medidas influenciadas por economia comportamental e a busca da "imunidade em massa" foram inicialmente encorajadas, com menor ênfase nas estratégias de distanciamento social. Acoplada a essa ideia, estavam iniciativas governamentais voltadas para a psicoeducação na mudança de comportamento, tais como disponíveis na plataforma da Behavioral Insight Team (vide www.bi.team), em parceria com o governo britânico. No entanto, dados epidemiológicos recentes desencorajaram essa estratégia, tendo em vista o impacto direto no sistema de saúde e o substancial risco para a população ${ }^{5}$. Novamente, as medidas de 
isolamento social passaram a figurar como as estratégias mais apropriadas para lidar com a pandemia.

Além dos impactos biológicos e econômicos, o novo coronavírus repercute significativamente na saúde mental, especialmente em razão do temor pela exposição ao contágio, ao adoecimento e à morte, pelas situações de quarentena e isolamento social. Esses aspectos tendem a intensificar sintomas de transtornos em saúde mental e produzir alterações subclínicas, principalmente em pessoas com histórico de problemas de saúde ou aquelas que estão na "linha de frente" na atenção à população, produzindo emergências subsequentes $2,6-8$.

Problemas acerca do enfrentamento da SARSCoV-2, identificados na China, país de origem da doença, são observados no Brasil, em que o foco nos cuidados biomédicos, em detrimento da saúde mental e da atenção psicossocial, prevalece', como pode ser verificado no Plano de Contingência da COVID19 elaborado pelo Governo do Distrito Federal (GDF, 2020). Após a epidemia da gripe provocada pelo vírus H5N1, em 2003, elaboraram-se diretrizes voltadas à vigilância epidemiológica, que delimitou uma rede estruturada para enfrentar, em 2009, a influenza H1N11, com foco, principalmente, no diagnóstico da doença e na preparação do cenário de isolamento e confinamento da COVID-19.

Situações ou ambientes de isolamento e confinamento $^{10}$, a exemplo dos necessários para conter a rápida evolução da crise do coronavírus, propiciam o aumento da preocupação com os impactos em saúde mental em curto e longo prazo ${ }^{4,7}$. $O$ confinamento, que implica na restrição de circulação das pessoas para evitar o potencial de crescimento de um contágio em forma de quarentena - termo utilizado primeiramente na Itália, em 1127 -, diferencia-se do isolamento no sentido em que este separa doentes de pessoas saudáveis ou sob suspeita, embora ambos os termos sejam utilizados como sinônimos durante epidemias ${ }^{2,11}$

$\mathrm{Na}$ China, desenvolveu-se um modelo de integração profissional online ${ }^{7}$, e a Comissão Nacional de Saúde do país asiático ${ }^{9}$ emitiu diretrizes para intervenções relacionadas à saúde mental, com medidas que liberam serviços online para pessoas com COVID-19 confirmada, aquelas com suspeita da doença, familiares e equipes de saúde ou de serviços fundamentais ${ }^{2}$, em regime de expedientes prolongados, em plantões ou prontidão 24 horas $^{12,13}$. Na Coréia do Sul, um sistema semelhante foi proposto ${ }^{4}$.

As recomendações no Brasil para atendimento de psicólogos online exigem cadastro e aprovação pelo Conselho Regional de Psicologia de cada estado, mediante diretrizes gerais do Conselho Federal de Psicologia (CFP). Diante da emergência de saúde pública com a COVID19, O CFP flexibilizou as diretrizes da Resolução $n^{\circ}$ 011/2018 durante os meses de março e abril de 2020, com possibilidades de prorrogação e novas alterações excepcionais, de modo a permitir o atendimento online mediante cadastro no sítio https://e-psi.cfp.org.br, porém sem necessidade de aguardar a aprovação para realizar o trabalho remoto via tecnologias ${ }^{14}$. No caso do Conselho Federal de Medicina (CFM), frente aos avanços da COVID-19 no Brasil, houve um posicionamento formal com relação à atuação de médicos quanto a alertas e orientações às autoridades e à população em geral sobre a epidemia, nesse esforço nacional ${ }^{15}$.

O CFP não recomenda, conforme o Artigo $6^{\circ}$ da Resolução nº 011/2018, atendimentos por psicólogos à distância em situações de emergência e desastres, a fim de evitar a vitimização, a patologização e a manipulação das pessoas envolvidas ${ }^{16,17}$. O CFP entende como cenário de emergências situações nas quais a pessoa se encontre em sofrimento psíquico grave, constituindo-se uma crise psicológica. Caso não seja possível atender de forma presencial, como na situação da pandemia, sugere-se atendimento online por um tempo delimitado, de modo a encaminhar a pessoa em crise aos dispositivos de cuidado do Sistema Único de Saúde (SUS) ${ }^{14}$, visando resguardar a integridade dos profissionais que devem evitar a exposição a agentes biológicos.

A recomendação da Sociedade Brasileira de Infectologia e da Associação Médica Brasileira (AMB, vide amb.org. br), atualizada em março de 2020, é de que somente procurem serviços de emergência pacientes com febre há mais de 48 horas, com falta de ar, piora progressiva de seu quadro gripal ou agravamento de sua doença de base, além de descompensação de doenças clínicas. Pacientes com resfriado ou com síndrome gripal podem ter COVID-19. Logo, todos precisam ser colocados em isolamento respiratório domiciliar por 14 dias. Outros vírus, como o rinovírus, podem causar resfriado e não 
são distinguíveis clinicamente, impossibilitando excluir a hipótese de COVID-19 sem o exame virológico. Contudo, os testes não serão realizados em todos os casos de resfriados e síndrome gripal, em virtude da escassez de kits diagnósticos.

O Ministério do Trabalho recomenda que trabalhadores que apresentem resfriado ou síndrome gripal devem ser afastados e permanecer em isolamento domiciliar por 14 dias, tendo direito a faltas abonadas, mesmo sem atestado médico, uma questão a ser refletida no que tange aos profissionais da saúde que estão na linha de frente atendendo pacientes com COVID-19. Os testes somente estão disponíveis para pacientes com a forma grave da doença, e um profissional de saúde que apresente resfriado ou síndrome gripal ficará afastado 14 dias em plena crise, caso não tenha acesso à confirmação.

A situação de confinamento e isolamento exige a resolução de outros problemas nunca antes considerados em dimensões territoriais de países com suas fronteiras fechadas. Situações anteriores de emergências, como deslizamentos de terra, permitiam o contato presencial do profissional de psicologia com as pessoas afetadas, de modo a possibilitar práticas que no cenário atual não são concebidas, como, por exemplo, atividades grupais. Entretanto, não somente psicólogos, mas médicos e outros servidores podem prestar primeiros atendimentos psicológicos (PAP) em cenários de emergências ${ }^{2,18-21}$, recomendados pela OMS, pois não constituem técnicas exclusivas de uma categoria.

Nas emergências, os psicólogos são requisitados para utilizarem técnicas de comunicação, psicoterapias breves, habilidades para auxiliar na resolução de problemas em situações de crises, no manejo e controle das emoções, na aplicação de instrumentos de mensuração, especialmente os de rastreio de comprometimentos psíquicos em versões breves, bem como na interpretação de resultados individuais, tendo em vista a prévia compreensão da complexidade do setting 13,19,21-23. A prestação de serviços aos profissionais de uma emergência deve pautar-se em avaliações objetivas e na abertura à reflexão, de forma a reconhecer as urgências e promover a human agency ${ }^{24}$, ou seja, a capacidade do profissional em intervir em cenários instáveis, com mais atenção e autonomia; de forma sintética, seguir três passos básicos: olhar, escutar e promover vínculo.
Além desse aspecto, é importante ressaltar que a maioria dos profissionais que atua em hospitais, que atende e atenderá um grande número de pacientes, não foi treinada para prestar assistência à saúde mental em situações de emergência de grande porte e tampouco está preparada para enfrentar a condição de confinamento por longos períodos, exigindo o seguimento de protocolos específicos em meio à probabilidade de pânico ou histeria coletiva ${ }^{2,7}$. O confinamento provocado pela epidemia resulta em alterações da rotina e do contato social e físico, observado em estudos de ambientes isolados, confinados e extremos (ICE) ${ }^{13,25,26}$. Nessa condição, acentua-se a manifestação de tédio, frustração e a sensação de isolamento ampliada, ou o denominado isolamento autônomo ${ }^{10}$, o medo de contaminação e outros receios e angústias relacionados à duração dos suprimentos, aos prejuízos econômicos, e no caso das equipes de saúde expostas ao risco mais extremo, a insatisfação com as provisões e recursos técnicos ${ }^{27}$.

É motivo de estresse, também para a população, o desencontro, a insuficiência e a incoerência de informações ${ }^{2,11}$. Algumas recomendações para o trato com as pessoas que apresentem resultado positivo para o vírus restringem a utilização de termos como "casos COVID-19" ou "doentes", definindo-as como "pessoas que têm COVID-19", "em tratamento" ou "se recuperando" de COVID-19, reduzindo o risco de estigma pela doença ${ }^{28}$. O CFM menciona a "importância do acesso à informação e à educação para mudança de comportamento e adoção de hábitos saudáveis", recomendando ainda "a realização de amplas campanhas de esclarecimento pelo Governo com foco na população em geral"15.

As emergências têm um caráter imediato e demandam a concentração de esforços para a resolução do problema-alvo $16,20,29,30$. Quanto mais rápidas as ações de resposta às emergências, menor o impacto, danos e prejuízos - já previstos para cenários mundiais ${ }^{31}$ - nas etapas definidas pela OMS: (a) etapa prévia, pré-crítica ou de prevenção; (b) etapa crítica ou da emergência propriamente dita; (c) período pós-crítico, pósemergências e de recuperação ${ }^{29,32}$. Médicos e psicólogos em território brasileiro, voluntários ou contratados para uma emergência devem estar atentos às diretrizes e orientações previstas no Plano Nacional de Gestão de 
Riscos e Resposta a Desastres Naturais, implementado em 2012 pelo Governo Federal, organizadas em cinco eixos: 1) prevenção (políticas habitacionais, direitos humanos, defesa civil nas escolas, formação para os profissionais da políticas públicas, planos de contingência) ${ }^{23}$; 2) mitigação (recursos estruturais); 3) preparação (mobilização comunitária, rotas de fuga, simulações de confinamento, etc.); 4) resposta (assistência); 5) reconstrução (ações com a finalidade de retornar às atividades de vida diária da população) ${ }^{33}$.

Defende-se a atuação de médicos e psicólogos na gestão integral de riscos de emergências e desastres. $\mathrm{Na}$ deflagração ou iminência de uma emergência, as respostas providas pelo Estado abarcam: o suporte à saúde médica e psicológica; abrigos/refúgios e condições sanitárias; alimentos e medicamentos para os afetados; além de recursos para as pessoas que prestam serviços essenciais ${ }^{19}$, como equipamentos de proteção individual (EPI) - máscaras, luvas, álcool em gel, etc.

Estratégias de gestão diferenciadas de atenção especializada em saúde mental devem se concentrar, especialmente, nos grupos de vulneráveis ${ }^{23}$ : idosos e crianças, mesmo que o percentual de infantes que desenvolvem a sintomatologia mais grave seja baixo. No caso da pandemia, pacientes com comorbidades prévias ou baixa imunidade, imigrantes e trabalhadores de serviços essenciais também devem ser objeto de atenção especial|2,734. No Brasil, deve-se ressaltar a necessidade de medidas dirigidas a pessoas em situação de rua, presidiários, comunidades periféricas e pouco assistidas pelo poder público e pelas comunicações em geral.

O autoisolamento de idosos, no Reino Unido, já consiste em uma recomendação planejada para os próximos meses em função da COVID-19, medida adotada também por outros países para não sobrecarregar os sistemas de saúde. Porém, tal isolamento acarreta um notório problema de saúde pública, já que os idosos requerem maiores cuidados para lidar com complicações comuns da faixa etária: cardiovasculares, neurocognitivas, autoimunes e de ordem mental, com destaque para o maior risco de depressão e ansiedade com as restrições de interações ${ }^{35,36}$.

As comunidades nas periferias das cidades têm altas densidades habitacionais, condições sanitárias e de moradia precárias, facilitando a rápida contaminação pelo coronavírus. As políticas públicas mal conseguem alcançar essas comunidades, deixando seus moradores vulneráveis a doenças altamente contagiosas ${ }^{37}$.

As preocupações quanto às prisões visam impedir uma proliferação desenfreada da epidemia, considerando que no país há superlotação, e o risco de infecções é elevado em um local mal ventilado, com condições sanitárias precárias e de contato próximo inevitável ${ }^{38}$. O histórico de surtos em presídios remonta ao registro da prisão de San Quentin, nos EUA, em 1918, problema que expõe a necessidade de libertar prisioneiros em todo o mundo para reduzir a transmissão acelerada do vírus desde o início de 2020 e, também, reduzir as chances de colapsar os serviços de atendimento aos afetados, que já não comportam as demandas cotidianas ${ }^{39}$. As medidas do governo têm como propósito retardar o pico da epidemia e, consequentemente, o colapso no sistema de saúde, que tem capacidade limitada para receber a população. Em algumas regiões, a capacidade do sistema de saúde para atender as demandas já era insuficiente antes da epidemia, e os casos de infartados, acidentados, etc., somam-se aos pacientes que buscam atendimento por sintomas da COVID-198.

A transmissibilidade da COVID-19 é elevada, embora diversas características da doença sejam desconhecidas, desde todas as formas de transmissão e sintomas - dor abdominal causada por adenite mesentérica, aumento dos linfonodos de forma dolorosa que pode até confundirse com apendicite, miocardites e encefalites, além dos clássicos sinais de gripe - até os impactos em internação grave e letalidade ${ }^{1}$. O distanciamento social, produto do confinamento e do isolamento, reduz o número de contaminados e, consequentemente, os casos graves de pessoas em grupos de risco que possam necessitar de unidades de tratamento intensivo (UTI), em quantidade restrita no SUS e na rede privada ${ }^{1,23}$.

Em um contexto normal, uma única pessoa pode contaminar 400 pessoas em 30 dias. Se as interações se reduzem em 75\%, a probabilidade é de que, em 30 dias, uma pessoa contamine menos de duas pessoas, logo, que haja uma lotação em níveis aceitáveis nos centros de atendimento ${ }^{40}$. A sobrecarga nos hospitais e demais serviços saúde, além dos grupos que atuam na limpeza, segurança e transporte, que não puderam ser paralisados, tende a ser ampliada pela apreensão de falta 


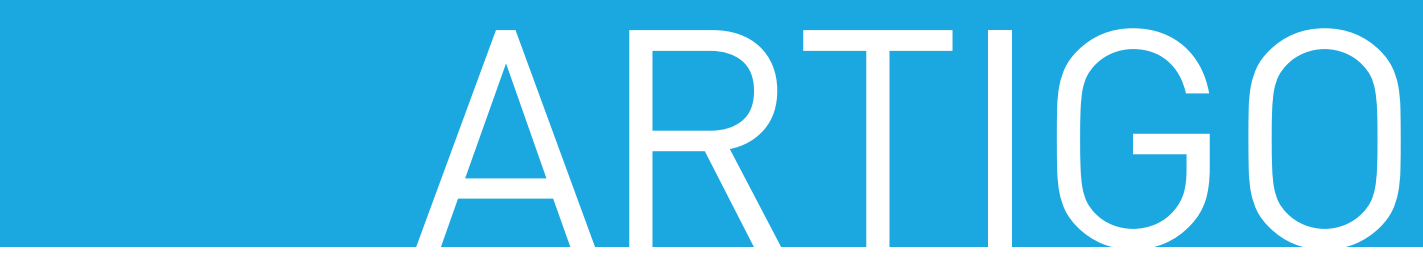

iminente de mantimentos, do aumento da criminalidade, da redução de acesso a atendimentos especializados e o temor do "fim do mundo"

As estimativas de um estudo com a COVID-19 na China indicam uma alta taxa de infecções não documentadas, acima de 80\%, e dessas infecções, ao menos 50\% com potencial de contágio, reforçando incentivos de mudança de comportamento (cobrir a boca ao tossir, distanciamento social e autoisolamento ao primeiro sinal suspeito) com o objetivo de interromper o avanço da epidemia em paralelo à redução de mobilidade humana ${ }^{41}$. $\mathrm{O}$ estudo de Li et al. ${ }^{41}$ apresentou resultados consistentes, que justificam a necessidade de investimentos que permitam um aumento radical tanto da identificação quanto do isolamento de pessoas com COVID-19 para o controle da pandemia, associados ao confinamento de indivíduos saudáveis e assintomáticos, esforços bem-sucedidos do governo chinês na queda da taxa de disseminação do vírus ${ }^{41}$.

A revisão de Brooks et al. ${ }^{11}$ apresenta dados significativos a respeito dos impactos psicológicos causados por pandemias. Em quarentenas impostas no passado, foram registrados casos de suicídio e agressividade extrema, além da manifestação de sintomas de estresse agudo poucos dias após a implementação da quarentena. Especialmente em equipes que trabalham em hospitais, registrou-se também exaustão, redução da empatia, ansiedade, irritabilidade, insônia e decaimento de funções cognitivas e do desempenho ${ }^{11}$.

Aqueles em quarentena, por estarem próximos de pacientes confirmados com a doença, relatam medo, nervosismo, tristeza e culpa ${ }^{4,11}$. Uma pesquisa realizada por Li et al. ${ }^{3}$ durante a pandemia da COVID-19 na China avaliou o trauma secundário em 214 pessoas do público geral, 234 enfermeiros da linha de frente e 292 enfermeiros que não lidaram diretamente com a COVID19. O trauma secundário foi significativamente maior no público geral e nos enfermeiros que não estavam na linha de frente em comparação com aqueles profissionais que lidam com a doença diretamente ${ }^{3}$. O número de profissionais da linha de frente contaminados pelo coronavírus cresce exponencialmente; somente na Itália, $20 \%$ dos trabalhadores foram infectados até o mês de março de 2020, entre eles alguns vieram a óbito ${ }^{42}$.

O enfrentamento da situação desencadeada pela COVID-19 fez o mundo assistir a milhões de pessoas em diversos países atendendo aos apelos para ficar em casa, a fim de minimizar os impactos da transmissão do vírus, ao mesmo tempo em que profissionais da saúde e de serviços essenciais mantinham e ampliavam o trabalho de assistência ${ }^{42}$. $O$ medo de contrair o vírus tornou-se companheiro dos dias de trabalho, mas o receio de leválo para casa e contaminar o ambiente dos familiares é motivo de reflexão sobre os riscos ocupacionais dessa profissão ${ }^{43}$.

Profissionais treinados para atender as necessidades imediatas dos pacientes ${ }^{44}$ trabalham diariamente em ambientes com o propósito da cura ou da redução de dor de enfermos, atividade que pode causar prejuízos à saúde mental ${ }^{45}$ mesmo em tempos sem a presença da COVID-19. São fatores de risco ocupacionais do trabalho da enfermagem as longas jornadas de trabalho, a baixa remuneração, a falta de reconhecimento profissional, a sobrecarga mental e física de trabalho, bem como o risco de contaminação ${ }^{46}$. Em situações como essa, além da multiplicação do vírus, aumenta o número de profissionais adoecidos física e mentalmente. Logo, cabe aos psicólogos alocados em serviços de saúde, in loco ou via tecnologias remotas, o atendimento emergencial de profissionais com crises de ansiedade, ataques de pânico, exaustão emocional, depressão, fadiga de compaixão, entre outros sintomas de transtornos em saúde mental.

A fadiga de compaixão é uma condição que merece atenção especial, uma vez que não só profissionais da saúde colocados na linha de frente do tratamento de traumas correm alto risco de desenvolvê-la, mas também é uma condição que gera outros agravos psicológicos. É descrita como a convergência do estresse traumático secundário - trauma vivido através da empatia com aqueles que estão em contato com vítimas - e do burnout - esgotamento cumulativo físico e mental causado pela capacidade reduzida de lidar com estressores do ambiente cotidiano ${ }^{3,47}$.

Entre as principais alterações que devem ser observadas por profissionais que possam oferecer auxílio especializado em psicologia, presencialmente ou, no caso da pandemia, por via online $e^{2,27}$, destacam-se: estresse agudo, luto, sintomas depressivos e ansiogênicos, distresse, propensão ao suicídio, comportamentos violentos, transtornos mentais comuns (TMC), abuso de substâncias psicoativas, psicose, predisposição 


\section{COVID-19}

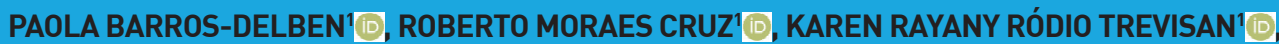
MARIA JULIA PEGORARO GAI'®D, RAQUEL VIEIRA COSTA DE CARVALHO'리, PEDRO AUGUSTO CROCCE CARLOTTO' ${ }^{10}$, ROBERTA BORGHETTI ALVES ${ }^{2}$ D, DANIELA SILVESTRE ${ }^{3}$ D. CRISTIANA ORNELLAS RENNER ${ }^{4} \mathbb{E}$, ANTONIO GERALDO DA SILVA ${ }^{5}$, LEANDRO FERNANDES MALLOY-DINIZ ${ }^{6} \mathbb{D}$

' Laboratório Fator Humano, Universidade Federal de Santa Catarina (UFSC). Florianópolis, SC. ${ }^{2}$ Universidade do Vale do Itajaí (UNIVALI), Itajaí, SC. ${ }^{3}$ Associação Brasileira de Medicina de Áreas Remotas (ABMAR), São Paulo, SP. ${ }^{4}$ CRX Psicologia, São Paulo, SP. ${ }^{5}$ Universidade do Porto, Porto, Portugal. Associação Brasileira de Psiquiatria (ABP), Rio de Janeiro, RJ, Brasil. Asociación Psiquiátrica de América Latina (APAL), Brasília, DF. ${ }^{6}$ Universidade Federal de Minas Gerais (UFMG), Belo Horizonte, MG. Universidade FUMEC, Belo Horizonte, MG.

ao pânico e estado de choque 2,4,22,28. Por outro lado, é importante levar em consideração a influência da satisfação de compaixão, no espectro positivo, um fenômeno associado à percepção de crescimento de sentimentos de bem-estar associados à ajuda efetiva prestada a outras pessoas. O estresse pós-traumático e a depressão severa figuram como consequência da quarentena, situação que prevalece por até 3 anos após o fim do sítio ${ }^{11}$.

Os sintomas principais da COVID-19, febre, tosse e hipóxia, associados aos sintomas do tratamento medicamentoso, que tende a provocar alterações do ciclo sono-vigília e ansiedade, em meio a um cenário de medo generalizado, contribuem para a ocorrência de sintomas de transtornos em saúde mental na maior parte dos envolvidos ${ }^{8}$. A COVID-19 produz impactos tão severos no organismo que é difícil diferenciar pacientes com câncer de pulmão e infectados ${ }^{34}$, e a disseminação desses resultados contribui para o pânico, inclusive entre os membros da equipe de saúde. Considerando as situações de confinamento e isolamento condicionados à pandemia, é saliente a necessidade de promoção de ações voltadas ao comportamento seguro, com destaque para o cumprimento de regras e ao autocuidado ${ }^{13,23,48}$.

O cumprimento de regras compreende uma ação com caráter prescrito na conduta coletiva e valorização social. São exemplos do cumprimento no cenário de pandemia: não sair de casa, exceto para atividades essenciais; lavar as mãos com água e sabão ou fazer uso de álcool em gel; cobrir a boca sempre que tossir ou espirrar; e evitar o contato próximo com pessoas de grupos de risco (idosos, pessoas com baixa imunidade, etc.). De maneira positiva, após a quarentena, muitos profissionais de saúde apresentaram comportamentos preventivos permanentes, minimizando o contato com possíveis contaminantes e seguindo os protocolos estabelecidos com maior engajamento e estado de vigilância pró-saúde ${ }^{11}$. Já o autocuidado é uma ação de sobrevivência que prioriza gerar ou manter o bem-estar pessoal: recorrer aos serviços de saúde, excepcionalmente durante a quarentena, somente ao perceber sintomas graves, conforme indicado pelo Ministério da Saúde, ou devido a outras condições que exijam atenção especializada de urgência, não eletivas; ingerir recursos básicos à autopreservação (água, alimentos, medicamentos, etc.); acessar condições em prol da higiene pessoal, da prática de exercícios físicos, do lazer, do convívio social e do entretenimento, de práticas religiosas ou mentais, do direito à privacidade, do direito a interações sociais (dentro do possível e atendendo às regras impostas pela quarentena de cada estado); e respeitar o ciclo descanso-expediente (sonovigília), mesmo em situação de home office ou, no caso dos serviços essenciais, em regime de plantão permanente ${ }^{13}$.

Um aspecto importante na atuação do profissional de saúde mental, frente às alterações de comportamento em pandemias, diz respeito à abordagem de estilos cognitivos da população em geral. Crenças e distorções cognitivas podem minimizar a adesão às recomendações de profissionais de saúde, diminuindo o efeito das iniciativas governamentais. Nesse sentido, Poland et al. ${ }^{49}$ destacam o papel do profissional de saúde na identificação dessas distorções cognitivas (ex.: negação de evidências científicas, crenças baseadas em teorias conspiratórias, generalizações excessivas, heurísticas e vieses) e do uso de intervenções para modificações de tais crenças e comportamentos, por meio de técnicas como a entrevista motivacional e outras.

A recomendação de ações de autocuidado tem como prerrogativa o gerenciamento de riscos para a redução da probabilidade de ocorrências de eventos indesejáveis ${ }^{23}$. Consideram-se como variáveis incontroláveis os atos inseguros, aspectos inerentes ao contexto e condutas que influenciam negativamente o sistema (erros, violações, subestimar ou ignorar riscos, negligência), assim como a percepção inicial de uma baixa incidência de contaminações ou número de mortes, o que favorece a uma falsa sensação de segurança e até mesmo instaura uma sensação de conspiração ${ }^{50}$. O Grupo Executivo Interministerial de Emergência em Saúde Pública de Importância Nacional e Internacional foi instaurado pelo Ministério da Saúde do Brasil no início de 2020 visando à construção de protocolos de ação e ao acompanhamento da pandemia da COVID-19¹ , permitindo a educação de prevenção a atos inseguros.

Em quarentena, as pessoas apresentam padrões irregulares do ciclo sono-vigília, dietas pobres, exercitamse menos, propiciando sobrepeso e decaimento do sistema imunológico; temem pela infecção, sofrem com a restrição social e espacial, com maior propensão a apresentarem estresse pós-traumático ${ }^{11,27}$. Logo, é 


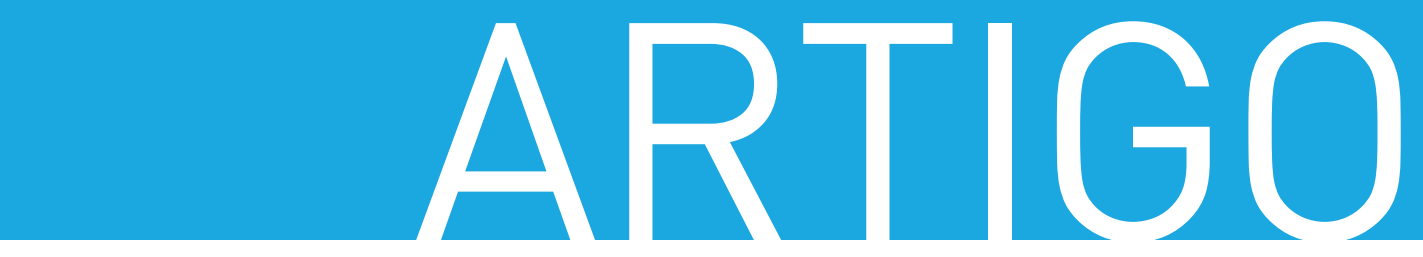

fundamental a atenção permanente para minar fake news, com notícias em linguagem acessível para cada grupo da sociedade e condicionamento de comportamentos em prol da saúde ${ }^{1}$. De forma sintetizada, algumas recomendações no âmbito da saúde mental voltada a profissionais e gestores em situações de confinamento e isolamento em virtude da epidemia da COVID-19 são:

1. Atenção a sintomas e sinais ansiogênicos, depressivos e de distresse - campanhas que auxiliem a identificá-los e orientem quanto ao que fazer nesses casos, com destaque para o ataque de pânico, prevenção do comportamento suicida (ênfase em pessoas idosas ou indivíduos que moram sozinhos - estimular o contato social por meio de tecnologias ou com vizinhos, mesmo que à distância) e de respostas emocionais alteradas frente à percepção de risco (informações sobre como melhorar a respiração e a concentração);

2. Estímulo à prática de atividades físicas, lazer, práticas de meditação ou religiosas, elaboração de rotinas de estudo e trabalho e espaço para descanso;

3. Redução do tempo de exposição a notícias, nas TVs e outras mídias, a respeito do coronavírus;

4. Incentivo do consumo racional de alimentos e de água no período crítico, tranquilizando a população quanto ao abastecimento, com plano de alcance às residências - em especial para as pessoas de baixa renda e com dificuldades de locomoção. Para essa população, também é importante o fornecimento de álcool em gel, especialmente nos locais em que há maior deficiência na estrutura de saneamento básico;

5. Fornecimento de transporte gratuito para quem precisa se deslocar;

6. Serviços de educação e orientações recorrentes à população sobre o comportamento de lavar as mãos, de cobrir a boca ao tossir ou espirrar e, também, quanto à higiene do sono - evitando decaimentos cognitivos e alterações emocionais ou do humor;

7. Restrição da comercialização de bebida alcoólica nos supermercados - as alterações provocadas por substâncias psicoativas tendem a elevar o clima de tensão e são usualmente estratégias disfuncionais (coping disfuncional), ou seja, recorrer ao consumo de álcool em situações de confinamento e isolamento, além de influenciar na redução da imunidade biológica;

8. Criação de um sistema de atendimento e contingenciamento de protestos e veiculação de fake news ou teorias conspiratórias, evitando a propagação de ideias que aumentem o medo e a insegurança e que podem levar ao pânico generalizado;

9. Garantia e divulgação da presença do policiamento (inclusive com o reforço das forças armadas) nas ruas, evitando saques e outros tipos de violência;

10. Campanha de cuidado com pessoas próximas - identificar voluntários que possam realizar o monitoramento e apoio a pessoas que moram sozinhas ou demonstrem algum tipo de restrição de movimento, para que possam auxiliar na realização de compras e na verificação do estado de saúde. Incentivo à solidariedade com as pessoas no momento de crise, algo que ajuda também o ajudante. Monitoramentos podem ser feitos à distância, e a assistência faz crescer vínculos na comunidade;

11. Suporte ao luto e situações de impedimentos de acompanhar falecidos em decorrência da epidemia, que, inclusive, não se deve limitar apenas nos momentos iniciais da crise.

A COVID-19 não faz distinção de etnia ou nacionalidade, e todos merecem suporte para enfrentar a situação ${ }^{28}$. No Brasil, os desafios atrelados às estratégias de mitigação dos riscos à saúde mental são evidentes, e emergem questões delicadas a serem enfrentadas, destacadas nas orientações do CFM e do CFP para a atuação dos profissionais. Destaca-se, nesse cenário, a busca de soluções relativas às desigualdades encontradas no país acerca do acesso à internet no período de confinamento e da familiaridade com plataformas digitais, tanto pelos profissionais de saúde, quanto pela população, com destaque para os grupos de risco: idosos, pessoas de baixa renda sem conexão, presidiários, pessoas em condição de rua, etc. Em nota orientativa, o Conselho Regional de Psicologia de Santa Catarina (CRP-SC) salienta a utilização do recurso telefônico como forma de promover o acolhimento às pessoas que não têm acesso à internet ${ }^{51}$. 
Um trabalho integrado entre os órgãos administrativos deve organizar ações para a resolução dos problemas apontados e planejar, em longo prazo, tanto a recuperação das pessoas atingidas, quanto o acompanhamento de casos ao longo da crise, baseando-se na racionalidade científica ${ }^{2,22,52}$. Considerando que os sintomas de transtornos em saúde mental podem perdurar por meses e até anos após a quarentena, as necessidades de planos de mitigação dessas consequências devem ser discutidas de imediato. Entre as estratégias possíveis, é relevante demonstrar respeito pela pandemia e suas consequências na saúde física e mental, assim como promover o autocuidado, seja por meio de tecnologias ou de ações coordenadas nas regiões de difícil acesso e menor condição econômica ${ }^{27}$.

Vale ressaltar que uma crise não é, em essência, caracterizada somente por seu aspecto negativo (restrições sociais, perda de autonomia, possível adoecimento). Uma crise é um evento que foge à norma e ao esperado, seja em função de sua intensidade ou prolongamento, e que transforma aquele que a vivencia. Uma forma de interpretar a crise é vê-la como uma oportunidade frente às mudanças autoimpostas. Em diversos sentidos, indivíduos e organizações públicas e privadas estão aprendendo a realizar atividades cotidianas e laborais em formatos diferenciados, o que possivelmente representará um marco histórico de organização social. Aspectos como o teletrabalho e o home office, que alteram a rotina familiar e doméstica, mas permitem o isolamento e reduzem a necessidade de tempo em deslocamento para locais físicos de trabalho, passam a fazer parte das possibilidades atuais para que atividades laborais continuem a ser realizadas.

Artigo submetido em 23/03/2020, aceito em 23/03/2020. Os autores informam não haver conflitos de interesse associados à publicação deste artigo.

Fontes de financiamento inexistentes.

Correspondência: Paola Barros-Delben, Laboratório Fator Humano, Bloco E, Centro de Filosofia e Ciências Humanas, Universidade Federal de Santa Catarina, Campus Reitor João David Ferreira Lima, $\mathrm{s} / \mathrm{n}^{\circ}$, Trindade, 88040-900, Florianópolis, SC. E-mail: p.barros.delben@gmail.com

\section{Referências}

1. Lana RM, Coelho FC, Gomes MFDC, Cruz OG, Bastos LS, Villela DAM, et al. Emergência do novo coronavírus (SARS-CoV-2) e o papel de uma vigilância nacional em saúde oportuna e efetiva. Cad Saude Publica. 2020;36:e00019620. Epub Mar 13, 2020

2. Qiu J, Shen B, Zhao M, Wang Z, Xie B, Xu Y. A nationwide survey of psychological distress among Chinese people in the COVID-19 epidemic: implications and policy recommendations. Gen Psychiatry. 2020;33:e100233.

3. Li W, Yang Y, Liu ZH, Zhao YJ, Zhang Q, Zhang L, et al. Progression of mental health services during the COVID-19 outbreak in China. Int J Biol Sci. 2020;16:1732-8.

4. Park SC, Park YC. Mental health care measures in response to the 2019 novel coronavirus outbreak in Korea. Psychiatry Investig. 2020;17:85-6.

5. WHO Collaborating Centre for Infectious Disease Modelling; MRC Centre for Global Infectious Disease Analysis; Abdul Latif Jameel Institute for Disease and Emergency Analytics; Imperial College London. Report 3: transmissibility of 2019-nCoV [Internet]. 2020 Jan 22-24 [cited 2020 Apr 6]. www.imperial.ac.uk/mrc-globalinfectious-disease-analysis/covid-19/report-3transmissibility-of-covid-19/

6. Bertolote JM, Mello-Santos Cd, Botega NJ. [Detecting suicide risk at psychiatric emergency services]. Braz J Psychiatry. 2010;32 Suppl. 2:S8795.

7. Lima CK, Carvalho PM, Lima IA, Nunes JV, Saraiva JS, de Souza RI, et al. The emotional impact of coronavirus 2019-Ncov (New Coronavirus Disease). Psychiatry Res. 2020;287:112915.

8. Xiang YT, Yang Y, Li W, Zhang L, Zhang Q, Cheung $\mathrm{T}$, et al. Timely mental health care for the 2019 novel coronavirus outbreak is urgently needed. Lancet Psychiatry. 2020;7:228-9.

9. Duan L, Zhu G. Psychological interventions for people affected by the COVID-19 epidemic. Lancet Psychiatry. 2020;7:300-2.

10. Barros-Delben P, Pereira GK, de Melo HM, Thieme AL, Cruz RM. Mapeamento de estressores 
no trabalho de expedicionários do Programa Antártico Brasileiro (PROANTAR). Psic Teor Pesq. 2019;35:e3539.

11. Brooks SK, Webster RK, Smith LE, Woodland L, Wessely S, Greenberg N, et al. The psychological impact of quarantine and how to reduce it: rapid review of the evidence. Lancet. 2020;395:91220.

12. Barros-Delben P, Cruz RM, de Melo HM, Teixeira ML, de Mendonça SA, Pereira GK, et al. Coping e padrões biológicos de sono em expedicionários antárticos. Rev Psicol Pesqui. 2019;13:147-68.

13. Cruz RM, Barros-Delben P. Avaliação psicológica de fatores humanos em ambientes isolados, confinados e extremos (ICE). In: Baptista MN, Muniz M, Reppold CT, da Silva Nunes CHS, Carvalho LF, Primi R, et al. Compêndio de Avaliação Psicológica. Petrópolis: Vozes; 2019. p. 200-10.

14. Conselho Federal de Psicologia (CFP). Nota orientativa às(aos) psicólogas(os): trabalho voluntário e publicidade em Psicologia, diante do Coronavírus (COVID-19) [Internet]. 2020 Mar 3 [cited 2020 Mar 23]. site.cfp.org.br/ nota-orientativa-asaos-psicologasos-trabalhovoluntario-e-publicidade-em-psicologia-diantedo-coronavirus-covid-19/

15. Conselho Federal de Medicina (CFM), Associação Nacional de Hospitais Privados (ANAHP). Posição do Conselho Federal de Medicina sobre a pandemia de COVID-19: contexto, análise de medidas e recomendações [Internet]. 2020 Mar 18 [cited 2020 Mar 23]. www.anahp.com.br/ noticias/noticias-anahp/posicao-do-conselhofederal-de-medicina-sobre-a-pandemia-decovid\%E2\% 80\%9019-contexto-analise-demedidas-e-recomendacoes/

16. Dário PP, Malagutti W. Desastres naturais: contribuições para atuação do psicólogo nos desastres hidrológicos. J Manag Prim Health Care. 2019;10:1-18.

17. Hodecker M, Alves RB. Intervenção em crise realizada com pessoas afetadas por emergências em uma Companhia de Bombeiros Militar. Est Inter Psicol. 2019;10:243-59.
18. de Albuquerque BS, Zacarias GM. A psicologia como aliada à gestão de risco em desastres. Rev Ordem Pub. 2016;9:109-20.

19. Paranhos ME, Werlang BSG. Psicologia nas emergências: uma nova prática a ser discutida. Psicol Cienc Prof. 2015;35:557-71.

20. Silva TLG, Mello PG, Silveira KAL, Wolffenbüttel L, Lobo BOM, Bicca CHM, et al. Primeiros Socorros Psicológicos: relato de intervenção em crise em Santa Maria. Rev Bras Psicoter. 2013;15:93-104.

21. Vasconcelos TP, Cury VE. Atenção psicológica em situações extremas: compreendendo a experiência de psicólogos. Psicol Cienc Prof. 2017;37:475-88.

22. Fonseca JP, Biasoto LGAP, Vicente RGV, Ramos RS, Padovan S. Intervenções psicológicas em emergências: a construção de uma práxis. In: Franco MHP. A intervenção psicológica em emergências: fundamentos para a prática. São Paulo: Summus; 2015. p. 61-104.

23. dos Santos PZ, Dias JI, Alves RB. Educação permanente sobre a atenção psicossocial em situação de desastres para agentes comunitários de saúde: um relato de experiência. Saude Debate. 2019:43:200-8.

24. Bandura A. Toward a psychology of human agency: pathways and reflections. Perspect Psychol Sci. 2018;13:130-6.

25. Palinkas LA. The psychology of isolated and confined environments. Understanding human behavior in Antarctica. Am Psychol. 2003;58:35363.

26. Tafforin C. Isolated and confined environments. In: Beysens DA, van Loon JJ. Generation and applications of extra-terrestrial environments on earth. Montrouge: River Publishers; 2015. p. 17381.

27. Wang G, Zhang Y, Zhao J, Zhang J, Jiang F. Mitigate the effects of home confinement on children during the COVID-19 outbreak. Lancet. 2020;395:945-7.

28. World Health Organization (WHO). Mental health and psychosocial considerations during COVID19 outbreak [Internet]. 2020 Mar 18 [cited 2020 Mar 23]. www.who.int/docs/default-source/ coronaviruse/mental-health-considerations.pdf 


\section{COVID-19}

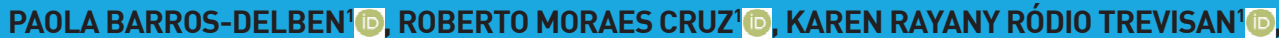
MARIA JULIA PEGORARO GAI'D, RAQUEL VIEIRA COSTA DE CARVALHO'미, PEDRO AUGUSTO CROCCE CARLOTTO'D, ROBERTA BORGHETTI ALVES² ${ }^{1}$, DANIELA SILVESTRE ${ }^{3}$, CRISTIANA ORNELLAS RENNER ${ }^{4} \mathbb{D}$, ANTONIO GERALDO DA SILVA ${ }^{5}$, LEANDRO FERNANDES MALLOY-DINIZ ${ }^{6} \mathbb{0}$

1 Laboratório Fator Humano, Universidade Federal de Santa Catarina (UFSC), Florianópolis, SC. ${ }^{2}$ Universidade do Vale do Itajaí (UNIVALI), Itajaí, SC. ${ }^{3}$ Associação Brasileira de Medicina de Áreas Remotas (ABMAR), São Paulo, SP. ${ }^{4}$ CRX Psicologia, São Paulo, SP. ${ }^{5}$ Universidade do Porto, Porto, Portugal. Associação Brasileira de Psiquiatria (ABP), Rio de Janeiro, RJ, Brasil. Asociación Psiquiátrica de América Latina (APAL), Brasília, DF. ${ }^{6}$ Universidade Federal de Minas Gerais (UFMG), Belo Horizonte, MG. Universidade FUMEC, Belo Horizonte, MG.

29. Durães UR, Santos JE dos. Emergencias, riesgos y desastres en Brasil: desafíos y experiencias de terapeutas ocupacionales. Rev Arg Ter Ocup. 2018;2:43:52.

30. Pacheco RF, de Souza SRE. A psicologia junto às políticas públicas em situações de emergência e desastres. Pretextos Rev Grad Psicol PUC Minas. 2017:2:131-49.

31. McKibbin WJ, Fernando R. The global macroeconomic impacts of COVID-19: seven scenarios [Internet]. 2020 Feb [cited 2020 Apr 6]. cama.crawford.anu.edu.au/sites/default/files/ publication/cama_crawford_anu_edu_au/202003/19_2020_mckibbin_fernando_0.pdf

32. Alves RB, Lacerda MAC, Legal EJ. A atuação do psicólogo diante dos desastres naturais: uma revisão. Psicol Estud. 2012;17:307-15.

33. Brasil, Governo Federal. Plano nacional de gestão de riscos e resposta a desastres naturais [Internet]. 2012-2014 [cited 2020 Apr 6]. pt.slideshare.net/ BlogDoPlanalto/plano-nacional-de-gesto-deriscos-e-resposta-a-desastres-naturais

34. Yang L, Xu HY, Wang Y. [Diagnostic and therapeutic strategies of lung cancer patients during the outbreak of 2019 novel coronavirus disease (COVID-19)]. Zhonghua Zhong Liu Za Zhi. 2020:42:E006.

35. Armitage R, Nellums LB. COVID-19 and the consequences of isolating the elderly. Lancet Public Health. 2020 Mar 19. pii:S2468-2667(20)30061-X. doi: 10.1016/S2468-2667(20)30061-X. [Epub ahead of print]

36. Chen X, Tian J, Li G, Li G. Initiation of a new infection control system for the COVID-19 outbreak. Lancet Infect Dis. 2020;20:397-8.

37. Edmundo K, Mello e Souza C, Carvalho ML, Paiva V. Vulnerabilidade ao HIV em favela do Rio de Janeiro: impacto de uma intervenção territorial. Rev Saude Publica. 2007;41 Suppl. 2:127-34.

38. World Health Organization (WHO), United Nations Office on Drugs and Crime (UNODC). Prisons and Health [Internet]. 2014 [coted 2020 Mar 23]. www.euro.who.int/_data/assets/pdf file/0005/249188/Prisons-and-Health.pdf
39. Kinner SA, Young JT, Snow K, Southalan L, LopezAcuña D, Ferreira-Borges $C$ et al. Prisons and custodial settings are part of a comprehensive response to COVID-19. Lancet Public Health. 2020;5:e188-e9.

40. Adhikari SP, Meng S, Wu YJ, Mao YP, Ye RX, Wang QZ, et al. Epidemiology, causes, clinical manifestation and diagnosis, prevention and control of coronavirus disease (COVID-19) during the early outbreak period: a scoping review. Infect Dis Poverty. 2020;9:29.

41. Li R, Pei S, Chen B, Song Y, Zhang T, Yang W, et al. Substantial undocumented infection facilitates the rapid dissemination of novel coronavirus (SARSCoV2). Science. 2020 Mar 16. pii: eabb3221. doi: 10.1126/science.abb3221. [Epub ahead of print]

42. The Lancet. COVID-19: protecting health-care workers. Editorial. Lancet. 2020;395:922.

43. Rocha ME, Freire KP, Dos Reis WPD, Vieira LTQ, De Sousa LM. Fatores que ocasionam o índice de transtornos depressivos e de ansiedade em profissionais de enfermagem: uma revisão bibliográfica. Braz J Develop. 2020;6:9288-9305.

44. Bare BG, Smeltezer SC. Tratado de enfermagem médico-cirúrgica. $9^{a}$ ed. Rio de Janeiro: Guanabara Koogan; 2002.

45. Carvalho MMMJ. A dor no estágio avançado das doenças. In: Angerami-Camon VA. Atualidades em psicologia da saúde. São Paulo: Pioneira Thomson Learrning; 2004. p. 85-101.

46. de Oliveira GF, Barbosa GA, de Souza LEC, da Costa CLP, Araújo RCR, Gouveia VV. Satisfação com a vida entre profissionais da saúde: correlatos demográficos e laborais. Rev Bioet. 2009;17:31934.

47. Cocker F, Joss N. Compassion fatigue among healthcare, emergency and community service workers: a systematic review. Int J Environ Res Public Health. 2016 Jun 22;13(6). pii: E618. doi: 10.3390/ijerph13060618.

48. Barros-Delben P, Cruz RM, Cardoso GM, Ariño DO, Pereira GK, Lopez M. Gerenciamento do comportamento seguro para manutenção da vida na Estação Antártica Brasileira. Rev Psicol Organ Trab. 2020;20:883-90. 


\section{ARTIGO DE REVISÃO}

PAOLA BARROS-DELBEN

ROBERTO MORAES CRUZ

KAREN RAYANY RÓDIO TREVISAN

MARIA JULIA PEGORARO GAI

RAQUEL VIEIRA COSTA DE CARVALHO

PEDRO AUGUSTO CROCCE CARLOTTO

ROBERTA BORGHETTI ALVES

DANIELA SILVESTRE

CRISTIANA ORNELLAS RENNER

ANTONIO GERALDO DA SILVA

LEANDRO FERNANDES MALLOY-DINIZ
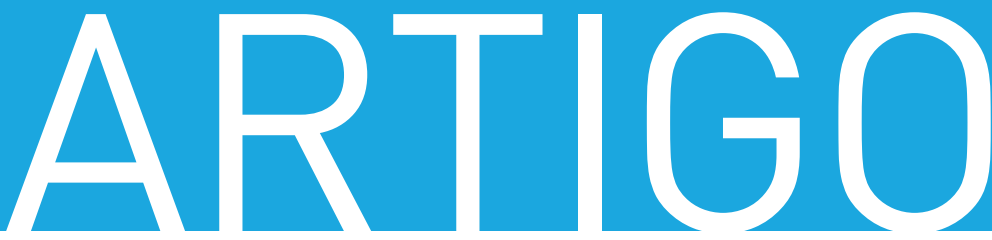

49. Poland GA, Fleming DM, Treanor JJ, Maraskovsky E, Luke TC, Ball EM, et al. New wisdom to defy an old enemy: summary from a scientific symposium at the 4th Influenza Vaccines for the World (IVW) 2012 Congress. 2013; 11 October, Valencia, Spain. Vaccine, 31, A1-A20.

50. Oliveira SG. Indenizações por acidente do trabalho ou doença ocupacional. $3^{a}$ ed. São Paulo: LTr; 2006.

51. Santa Catarina, Conselho Regional de Psicologia (CRP), $12^{a}$ Região. Orientação sobre a atuação da(o) psicóloga(o) diante do COVID-19 [Internet]. 2020 Mar 20 [cited 2020 Mar 23]. www.crpsc. org.br/noticias/orientacao-sobre-a-atuacao-dao-psicologa-o-diante-do-covid-19

52. Braga APA, Martins-Silva PO, Avellar LZ, Tristão KG, Ribeiro Neto PM. Produção científica sobre psicologia dos desastres: uma revisão da literatura nacional. Estud Psicol (Natal). 2018;23:179-88. 